It is known that a quintic surface having for double curve a proper conic or a pair of intersecting lines, but possessing no other singularities, is not rational. If we write the equation of a quintic having two intersecting double lines and then let the parameter which expresses the angle between them be zero, we obtain a surface of class 36 which is in general not rational, but which has four pinch points and three generally distinct triple points on the coincident double lines. It may be shown that just five relations must obtain among the coefficients of such an equation if it is to represent a rational surface of the type just discussed.

The University of California

\title{
AMOUNTS OF INVESTMENTS AT ANY NUMBER OF RATES OF INTEREST*
}

\author{
BY C. H. FORSYTH
}

The writer developed certain generalizations of the most common formulas of the mathematical theory of finance in the June-July, 1921, issue of this Bulletin. These generalizations were based upon the use of two and three rates of interest, instead of one.

It is the purpose of this short paper to extend some of these generalizations to the use of any number of rates of interest. In developing such generalizations it will be found that solutions including reasonably complete treatments of the various possible relative frequencies of conversions of the various rates of interest, while not unduly difficult, would be so complicated in form and be so monopolized by terms due only to those treatments that it would be very difficult to sift out the information which we most desire now, namely, the nature of the function itself when the number of rates

\footnotetext{
* Presented to the Society, October 29, 1927.
} 
is increased markedly. We shall therefore omit these objectionable terms by assuming that the period of conversion is the same for all rates of interest.

We shall denote the rate of interest realized directly from the principal by $i_{1}$. If then these interest payments are accumulated at a second rate $i_{2}$ the amount of an investment of one unit of principal by the end of $n$ conversion periods will be $1+i_{1} s_{\bar{n} \mid}$ where $s_{\bar{n} \mid}$ is to be valued at rate $i_{2}$. This, then, is the amount of a principal of one unit at two rates of interest.

If three rates of interest are used, the corresponding amount is the one unit plus the sum of the accumulations of the $n$ payments of $i_{1}$, where each of these accumulations is given by $i_{1}\left(1+i_{2} s_{\bar{n} \mid}\right)$ and $n$ has the successive values $n-1$, $n-2, \cdots, 2,1,0 ; s_{\bar{n} \mid}$ is to be valued at rate $i_{3}$. The amount is easily found and can be expressed as

$$
1+{ }_{n} C_{1} i_{1}+\frac{i_{1} i_{2}}{i_{3}}\left(s_{\bar{n} \mid}-{ }_{n} C_{1}\right),
$$

where ${ }_{n} C_{1}$ is the usual symbol for "the number of combinations, etc."

The procedure just illustrated is the same for all successive steps; the formula for $r$ rates of interest is used to accumulate the interest payments direct from the principal to give the formula for $r+1$ rates of interest. As the formula for $r+1$ rates is of the same form as that for $r$ rates, the formula is established automatically by induction as it is derived. The formula for the amount of an investment of one unit of principal for $n$ conversion periods, at $r$ rates of interest, may be written:

$$
\begin{aligned}
& 1+{ }_{n} C_{1} i_{1}+{ }_{n} C_{2} i_{1} i_{2}+\cdots+{ }_{n} C_{r-2} i_{1} i_{2} \cdots i_{r-2} \\
& \quad+\frac{i_{1} i_{2} \cdots i_{r-1}}{i_{r}{ }_{r-2}}\left(s_{\bar{n} \mid}-{ }_{n} C_{1}-{ }_{n} C_{2} i_{r}-\cdots-{ }_{n} C_{r-2} i_{r}{ }^{r-3}\right)
\end{aligned}
$$

$\left(s_{\bar{n} \mid}\right.$ to be valued at rate $\left.i_{r}\right)$.

The reader may find it of interest to see how the successive 
cases "nestle." Thus, if $i_{r}$ be replaced by $i_{r-1}$ in the formula given above, the reduction of that formula to the one for $r-1$ rates is easily followed.

For those who would care to check the summations leading to the formula given above it may be of help to note that $\sum s_{\bar{x} \mid}$ from $x=n-1$ to 0 inclusive is $\left(s_{\bar{n} \mid}-{ }_{n} C_{1}\right) \div i$ and that $\sum_{x} C_{r}$ from $n-1$ to 0 inclusive is ${ }_{n} C_{r+1}$. Evidently, the subscripts of the rates are all to be shifted one unit for each set of summations.

The formula for the amount of an annuity of one unit, at the end of each conversion period, for $n$ conversion periods, at $r$ rates of interest, would be obtained by accumulating each payment by the formula obtained above. The desired formula would be given then by the finite integral or sum of the formula given above, from $n-1$ to 0 inclusive. Evidently, this set of summations involves no shift in the subscripts of the rates of interest. The formula for the amount of such an annuity (at rate $i_{r}$ ) becomes

$$
\begin{aligned}
{ }_{n} C_{1} & +{ }_{n} C_{2} i_{1}+\cdots+{ }_{n} C_{r-1} i_{1} i_{2} \cdots i_{r-2} \\
& +\frac{i_{1} i_{2} \cdots i_{r-1}}{i_{r}{ }^{r-1}}\left(s_{\bar{n} \mid}-{ }_{n} C_{1}-{ }_{n} C_{2} i_{r}-\cdots-{ }_{n} C_{r-1} i_{r}{ }^{r-2}\right) .
\end{aligned}
$$

Attention was called, in the paper cited at the beginning, to the fact that the present value of an annuity at several rates of interest is indeterminate or meaningless unless the term "present value" of the annuity is defined more fully than usual and even then any such definition will refer to only one of several possible interpretations. 